\title{
La apuesta por la filosofía en el Instituto Mártires 20 de Febrero Secundaria: una retrospectiva ${ }^{1}$
}

\section{Betting on philosophy in the Instituto Mártires 20 de Febrero Secundaria: a retrospective}

DOI: $10.46932 / \mathrm{sfjdv2n2-061}$

Received in: january 1st, 2021

Accepted in: March 30th, 2021

\section{Cuitláhuac Rodríguez Campos}

: Licenciado en Filosofía por la Universidad Autónoma de Nayarit (2011), Maestro en Estudios Filosóficos por la Universidad de Guadalajara (2015) y Doctorando en Lógica y Filosofía de la Ciencia por la Universidad de Salamanca

Institución: Instituto Mártires 20 de Febrero Secundaria de la Universidad Autónoma de Nayarit. Dirección Institucional: Cd. de la Cultura “Amado Nervo", sin número; Edificio Secundaria Mártires 20 de Febrero, entre Unidad Académica Preparatoria No. 1 y CENDI; Colonia Menchaca.

Correo electrónico: cuiroca@uan.edu.mx

\section{RESUMEN}

En el presente texto se da a conocer cómo ha sido el proceso de la apuesta por la filosofía en el Instituto Mártires 20 de Febrero Secundaria, ante la problemática de la carencia de competencias por parte de los alumnos. El recuento incluye lo que ha sido la justificación de la introducción de las asignaturas filosóficas en los documentos rectores de la educación; el objetivo del enfoque de las distintas disciplinas filosóficas, y algunas de las dificultades y logros que se han tenido al apostar por la filosofía, junto con el resto de las asignaturas, como generadora de competencias en los alumnos. Así también, se da a conocer qué se está trabajando y qué falta por hacer.

Palabras clave: Educación Secundaria, Competencias, Filosofía, Ética, Lógica, Teoría de la Argumentación.

\begin{abstract}
In this text is made known how was the process of betting on philosophy in the Instituto Mártires 20 de Febrero Secundaria facing the problems related with the lack of competencies from students. The count includes the justification of the introduction of the philosophical matters in the governing documents of education; the aim of the different philosophic disciplines approach and some difficulties and achievements earned betting on philosophy, together with the rest of subjects, as competencies generator with the students. Also, is made known the way work is being done and what needs to be done.
\end{abstract}

Keywords: Secondary School, Competencies, Philosophy, Ethics, Logic, Argumentation Theory.

\footnotetext{
${ }^{1}$ Una primera aproximación a lo aquí tratado fue presentada en Pedagogía 2019. Encuentro Internacional por la Unidad de los Educadores, celebrado en el Palacio de Convenciones de La Habana, Cuba, del 4 al 8 de febrero del 2019. La presentación tuvo por título Tres ciclos escolares de experiencias ante la filosofía. El caso del Instituto Mártires 20 de Febrero Secundaria.
} 


\section{Introducción}

El dotar de competencias a los estudiantes de educación básica, que les permitan desempeñarse de manera eficiente en los niveles educativos posteriores, es una grave problemática, tal como se observa en los informes del Instituto Nacional para la Evaluación de la Educación (INEE), con los resultados desfavorables por parte de los alumnos próximos a finalizar la educación secundaria (2015 y 2016) . Esta problemática tiene como resultado la deficiencia que se observa en los estudiantes de los niveles medio superior y superior, al afrontar las situaciones académicas que en esos niveles educativos se les presentan. Por ello, es importante reflexionar sobre la problemática de adquisición de competencias en educación básica, especialmente en educación secundaria, y las competencias necesarias en el nivel universitario (Silva, 2016).

Teniendo en cuenta este antecedente, en el ciclo escolar 2015 -2016, el Instituto Mártires 20 de Febrero Secundaria abre sus puertas, impartiendo educación secundaria a los hijos de los trabajadores universitarios de la Universidad Autónoma de Nayarit, e introduce asignaturas filosóficas en su proyecto educativo: Ética, Lógica y Teoría de la Argumentación, con el objetivo, en conjunto con el resto de las asignaturas, de atacar la problemática de adquisición de competencias presente en educación secundaria.

En la exposición, se inicia mencionando algunos elementos que permitan situar el antecedente de fundación del Instituto Mártires 20 de Febrero Secundaria, así como la infraestructura del mismo; posterior a ello, se continúa mencionando cómo se llevó a cabo la selección, introducción y justificación de las disciplinas filosóficas en cada grado y ciclo escolar; se mencionan algunos de los obstáculos y logros presentes a lo largo de cuatro ciclos escolares, desde el ciclo escolar 2015 - 2016 al ciclo escolar 2018 - 2019, y se finaliza con algunas conclusiones y dando a conocer dónde estamos y qué falta por hacer.

\section{CONTEXTO}

El Instituto Mártires 20 de Febrero Secundaria (en adelante IM20FS) es una institución ubicada en Tepic, Nayarit, México, y ofrece educación secundaria a los hijos de los trabajadores universitarios; fue fundado en el año 2015, y tiene la característica especial de contar con una clave subsidiada: 18SES0029W. Esta prestación contractual para los trabajadores universitarios se establece en la cláusula 77 Bis del Contrato Colectivo de Trabajo del Sindicato de Empleados y Trabajadores de la Universidad Autónoma de Nayarit (SETUAN) (UAN y SETUAN, 2018).

El IM20FS se ubica dentro del campus de la Universidad Autónoma de Nayarit (UAN), y surgió gracias al esfuerzo realizado por el SETUAN, a través de la Asociación Civil Instituto Mártires 20 de 
Febrero A. C.; así como a las aportaciones económicas realizadas por los trabajadores agremiados al mencionado sindicado, las cuales permitieron la edificación del Instituto.

En cuanto a su infraestructura y organización se refiere, el IM20FS cuenta con: i) 9 aulas, tres para cada grado escolar, cada una con capacidad para 25 alumnos; ii) servicios sanitarios; iii) un aula que cuenta con 35 equipos de cómputo; iv) un laboratorio de ciencias, medianamente equipado; v) áreas deportivas: una cancha de fútbol, una cancha de voleibol y una cancha mixta (que cuenta con techumbre desde finales del ciclo escolar 2016 - 2017), tanto para basquetbol como para fútbol rápido, que también funge como espacio para la realización de las actividades cívicas; vi) área de enfermería; vii) área de psicología; viii) dos aulas para cuestiones administrativas, incluida dirección escolar y espacio para los docentes; ix) un área de comedor, que no es fija, y x) una cocina (cafetería), debido a que los alumnos tienen un espacio asignado para ingerir sus alimentos, tanto desayuno como comida, puesto que el horario escolar es de 07:40 h a 15:40 h. Y desde el ciclo escolar 2017 - 2018 es una escuela de organización completa.

Dado que se trata de una prestación para los trabajadores universitarios, se deriva que la comunidad estudiantil que se atiende, en lo correspondiente al nivel socioeconómico, es muy diversa. Los alumnos pueden ser hijos de trabajadores administrativos o manuales: intendencia, mantenimiento, secretariado, entre otros; o hijos de personal académico: docentes de asignatura en nivel medio superior o superior, investigadores, directivos, secretarios, etc. De igual manera, el nivel de escolaridad de los padres de familia también es diverso.

\section{LA APUESTA POR LA FILOSOFÍA}

La introducción de la filosofía en el IM20FS se justificó, en un primer momento, en la reforma realizada al artículo 3o. constitucional, en lo establecido en los incisos $c$ y $d$ de la fracción II:

c) [La educación] Contribuirá a la mejor convivencia humana, a fin de fortalecer el aprecio y respeto por la diversidad cultural, la dignidad de la persona, la integridad de la familia, la convicción del interés general de la sociedad, los ideales de fraternidad e igualdad de derechos de todos, evitando los privilegios de razas, de religión, de grupos, de sexos o de individuos, y

d) Será de calidad, con base en el mejoramiento constante y el máximo logro académico de los educandos (DOF, 2013).

Y, como bien se sabe, ya desde la Antigüedad, se aprecia el interés de los filósofos por formar jóvenes que reflexionan, que cuestionan, que se interesan en la búsqueda del conocimiento, que ejercen 
su libertad ${ }^{2}$. El interés de la filosofía radica en contribuir a la formación del ciudadano que vive en armonía consigo mismo, con los otros y con la sociedad; que desarrolla y reconoce los valores que propician el bien común; que adquiere competencias, de las cuales se hace consciente, que le permiten continuar aprendiendo, y que se conduce mediante la razón a lo largo de su vida y en contra de cualquier adoctrinamiento. Porque, tal como lo menciona Matsuura,

[...] la filosofía implica el ejercicio de la libertad gracias a la reflexión. Se trata, por ende, de juzgar sobre la base de la razón y no de expresar meras opiniones, no solo de saber sino también de comprender el sentido y los principios del saber, de actuar para desarrollar el sentido crítico, baluarte por excelencia contra toda forma de pasión doctrinaria (UNESCO, 2011, p. ix).

Es así que, la introducción de la filosofía se justificó tomando en cuenta la finalidad de la educación establecida en el artículo 3o. constitucional, el interés de la filosofía en la formación de ciudadanos y la misión del IM20FS, que consiste en:

[El compromiso con] [...] el desarrollo de habilidades, conocimientos, actitudes y valores, que se conviertan en competencias para la vida de nuestros alumnos; [en ser] una Institución que fomenta y valora el trabajo en equipo, así como el trabajo individual de sus alumnos; una Institución preocupada por fomentar el respeto y conservación del medio ambiente en sus alumnos, que promueve proyectos sustentables; una Institución que desarrolla las capacidades de los alumnos a través del pensamiento responsable y crítico, con una enseñanza activa, innovadora e integral, realizando una alianza de trabajo colaborativo entre maestros, padres y alumnos, y fomentando una conciencia ética y global en su comunidad estudiantil (IM20FS, 2019, p. 1).

También, se tuvo presente que dicha inclusión de la filosofía no iba en contra de las regulaciones para el funcionamiento de las Escuelas Secundarias (DOF, 1982).

\subsection{CICLO ESCOLAR $2015-2016$}

En el ciclo escolar 2015 - 2016 se da inicio al proyecto educativo del IM20FS, incluyendo a la filosofía en su currículo. Con esto, se da continuidad a la propuesta educativa llevada a cabo en la Primaria Mártires 20 de Febrero (PM20F) ${ }^{3}$. Este ciclo escolar se inició con una población de 70 alumnos en primer, y único, grado; en su mayoría egresados de la primera generación de la PM20F. La asignatura de Filosofía I: ética se introdujo en este grado escolar, dando así inicio al quehacer de la filosofía en el IM20FS.

Las razones que llevaron a la elección de introducir la asignatura de Filosofía I: ética en primer grado fueron dos: 1) atendiendo el plan de estudios, la asignatura de Formación Cívica y Ética solo se impartía en segundo y tercer grado de secundaria (SEP, 2011), y 2) dados los objetivos del IM20FS, por contribuir a la formación de los alumnos, se observó la necesidad que desde el primer grado escolar

\footnotetext{
${ }^{2}$ Recordemos la figura de Sócrates, cuyo interés en la formación de los jóvenes le valió ser condenado a muerte (Platón, 1985).

${ }^{3}$ Este instituto imparte educación primaria para los hijos de los trabajares universitarios, desde el ciclo escolar 2009 - 2010, donde el programa de Filosofía para Niños de Mathew Lipman forma parte del currículo.
} 
hubiera una asignatura enfocada, en conjunto con el resto de las asignaturas curriculares, en desarrollar competencias en los alumnos correspondientes al campo de formación de Desarrollo personal y para la convivencia.

La selección de las temáticas, que se tratarían en la asignatura de Filosofía I: ética, estuvo basada en contribuir al logro de los objetivos del IM20FS antes mencionados y atendiendo a los principios pedagógicos, principalmente el principio de incorporar temas de relevancia social: "Los temas de relevancia social se derivan de los retos de una sociedad que cambia constantemente y requiere que todos sus integrantes actúen con responsabilidad ante el medio natural y social, y la diversidad social, cultural y lingüística" (SEP, 2011, p. 36).

Por ello, se eligieron temas cuya finalidad era hacer pensar a los estudiantes sobre situaciones cotidianas, que les permitieran realizar un ejercicio de análisis y reflexión; que se dieran cuenta de lo problemático que son los temas y situaciones cotidianas al ponerles un poco de atención y cuestionarse sobre ellos, y que fueran conscientes de su propio proceso de pensamiento.

Entre los temas abordados se encontraban: ¿es correcto mentir?, ¿tienen derecho los animales no humanos?, la importancia de las preguntas, ¿cómo debemos actuar?, ¿qué es la ética?, ¿qué es la justicia?, ¿qué es un dilema ético?, ¿qué es la moral?, la muerte, el holocausto judío, aborto, trasplantes, entre otros (Collingwood, 1954; Estanislao, Álvarez y Guerra, 2015; Platón 1988; Tugendhat, Vicuña y López, 2010). Algunos de los casos abordados, que pertenecen a la bioética, fueron: Bebé Theresa, Siamesas Jodie y Mary y Tracy Latimer (Rachels, 2006). Así también, Willowbrook, Brophy, Baby M, Tatiana Tarasoff, no revelar un cáncer de próstata y solicitud de un hijo de ocultar información (Beauchamp y Childress, 1999).

El abordaje de los temas anteriores, cuyo espacio de impartición consistía en 2 módulos de 50 minutos por semana, permitió invitar a los estudiantes a determinar las preguntas importantes que se encontraban implícitas, determinar la idea central que se expresaba y determinar la problemática presente en el tema abordado. También, permitió invitar a los estudiantes a pensar sobre las siguientes preguntas: ante la problemática abordada ¿cómo actuarían ustedes?, ¿cómo determinar que una manera de proceder es correcta?, ¿cuál es el criterio?; ¿qué regla moral está involucrada en la problemática abordada?, ¿alguna vez han estado en situaciones similares a la presentada en la lectura?, de ser afirmativo, ¿cuál fue el criterio que utilizaron para actuar de determinada manera?

El enfoque con el que los temas fueron abordados tenían una finalidad: sacar a los estudiantes de su zona de confort y colocarlos, en la medida de lo posible, en situaciones que los forzaran, y encantaran, a pensar sobre las causas y consecuencias que se pudieran extraer del tema tratado; que les impulsaran a reflexionar y darse cuenta de las problemáticas existentes en la cotidianeidad. 
Este reflexionar y darse cuenta de las problemáticas, poco a poco permitió a los alumnos el formular sus aportaciones cada vez más puntuales y claras; así como a ser empáticos en las problemáticas que atañen a la sociedad. Esta tarea no resultó cosa fácil, pues el ser cuestionado genera conflicto. Las dificultades que se presentaron, serán mencionadas posteriormente.

\subsection{CICLO ESCOLAR 2016 - 2017}

La publicación del documento El Modelo Educativo 2016. El planteamiento pedagógico de la Reforma Educativa reafirmó la justificación de la introducción de la asignatura de Filosofía I: ética, puesto que el objetivo de la asignatura era consecuente a la orientación educativa establecida en el mencionado documento:

Educar a partir de valores implica formar a todos en el respeto a lo diverso, en el aprecio por la dignidad humana, en el rechazo a la discriminación y en las prácticas que promueven la solidaridad [...] además de formar individuos que aprecien y respeten la diversidad y rechacen toda forma de discriminación, es preciso que aprendan a reconocerse a sí mismos como personas que actúan en lo local y a la vez forman parte de una sociedad universal y habitan un planeta cuya preservación es responsabilidad de todos [...] (SEP, 2016, pp. 37-38).

Y permitió justificar la introducción de la asignatura de Filosofía II: lógica en el segundo grado escolar; así como trabajar en la planeación para introducir la asignatura de Filosofía III: argumentación en el tercer grado escolar, una vez llegado el momento. Lo anterior debido a que: "El desarrollo de las capacidades de pensamiento crítico, análisis, razonamiento lógico y argumentación son indispensables para un aprendizaje profundo que permita trasladarnos a las diversas situaciones para resolver nuevos problemas" (SEP, 2016, p. 13). Y, además,

La función de la escuela ya no es enseñar a los niños y jóvenes lo que no saben, sino aquello que necesitan para aprender a aprender. Se requiere una formación que permita a las personas adaptarse a nuevas situaciones mediante un proceso de educación permanente, que les asegure apropiarse de conocimientos y competencias para desplegar su potencial a lo largo de su vida (SEP, 2016, p. 39).

Con esto, en el ciclo escolar 2016 - 2017, la asignatura de Filosofía II: lógica se agrega al currículo del IM20FS. La comunidad estudiantil crece con el ingreso de 70 nuevos alumnos.

En esta ocasión, la selección de los temas de la asignatura estuvo orientada a subsanar determinadas carencias académicas observadas en los alumnos, por ejemplo: i) problemas de identificación de los elementos mínimos que componen una oración; ii) problemas para identificar los tipos de oraciones; iii) desconocimiento de lo que un concepto es, así como lo que una definición es y los tipos de ésta; iv) problemas para realizar inferencias correctas, y v) poca habilidad en los procesos formales.

A lo largo del curso, que también se impartió en dos módulos de 50 minutos por semana, se trabajaron temas como: concepto: extensión y comprehensión; definición: etimológica y real, así como 
las reglas de la definición, y tipos de oraciones (Escobedo, 1995). ¿Qué es la lógica? (Ferrater, 1957; Morado, 1999). ¿Qué es una inferencia?; ¿qué es deducción?; ¿para qué sirve la lógica?; principios lógicos; contradicción, tautología y contingencia; reglas de inferencia, y métodos de decisión (Amor 2003; Campirán, 2003; Deaño, 1985; Garrido, 1989; Garrido, 2001; Katayama, 2011; Lozano y Pérez, 2016). Autorreferencia y ¿qué podemos inferir? (Fernández y Villegas, 2011; Suppes, 1973).

Tras el abordaje y explicación de los diversos temas, se invitaba a los alumnos a realizar ejercicios que les permitieran fortalecer los aprendizajes y las herramientas adquiridas. El énfasis en la realización de los ejercicios fue constante, puesto que estos posibilitarían a los alumnos el apropiarse de las herramientas ofrecidas en esta asignatura.

El objetivo perseguido en la asignatura de Filosofía II: lógica fue cooperar en el desarrollo de habilidades de análisis y síntesis; de conceptualización y manejo de definiciones, y de habilidades inferenciales. Así también, familiarizar a los alumnos en cuestiones relacionadas al lenguaje objeto (uso) y metalenguaje (mención), lo mismo que con la noción de autorreferencia.

\subsection{CICLO ESCOLAR $2017-2018$}

La publicación de los documentos Carta sobre los fines de la educación en el siglo XXI, en donde se menciona qué tipos de mexicanos se desea formar, especificando el perfil de egreso deseado (SEP, 2017a) y Aprendizajes clave para la educación integral. Plan y programas de estudio para la educación básica, en el cual se establecen los Ámbitos de la Autonomía Curricular (SEP, 2017b), fue una razón más que permitió la justificación de la introducción de la filosofía en el IM20FS. Esto fue así, debido a la compatibilidad del perfil de egreso deseado y el objetivo de la filosofía en el IM20FS; además, abrió la posibilidad de que las asignaturas filosóficas impartidas se adecuaran en alguno de los Ámbitos de la Autonomía Curricular, siendo así reconocidas por las autoridades educativas.

En el ciclo escolar 2017 - 2018, la matrícula escolar aumentó con el ingreso de 75 alumnos y, como se estuvo planeando, la asignatura de Filosofía III: argumentación formó parte del currículo, siendo impartida a los alumnos de tercer grado, con el mismo espacio de módulos que el resto de las asignaturas filosóficas. Con esto, se precisó la introducción de la filosofía en el proyecto educativo del IM20FS y la concordancia con el Plan de estudios, puesto que en el ámbito educativo

[...] resulta necesario formar al individuo para que sea capaz de adaptarse a los entornos cambiantes y diversos, maneje información de una variedad de fuentes impresas y digitales, desarrolle un pensamiento complejo, crítico, creativo, reflexivo y flexible, resuelva problemas de forma innovadora en colaboración con otros, establezca metas personales y diseñe estrategias para alcanzarlas (SEP, 2017b, p. 25). 
La selección de los temas a impartir en la asignatura de Filosofía III: argumentación se basó en desarrollar competencias en lectura y escritura, en miras de que los alumnos expresaran sus ideas de forma ordenada, clara y argumentada. Por ello, los temas que se incluyeron fueron: ¿qué es un argumento?, ¿para qué argumentar?, ¿tiene alguna utilidad argumentar? (Vega, 2016; Weston, 2013). ¿Qué es una falacia? y ejemplos de falacias (Herrera y Torres, 2007; Schulman, s/f; Vega, 2013). ¿Es posible estructurar una argumentación?, ¿todas las razones son igual de fuertes?, ¿qué es un punto de vista?, razones en favor de un punto de vista y esquemas argumentativos (Eemeren y otros, 2006; Marraud, 2013). Por último, ¿qué es un ensayo? y normas APA (Centro Javeriano de Escritura, 2019; Oscos, 2010a y 2010b; Pacheco, 2006; Silva y Juárez, 2013).

El objetivo de la asignatura de Filosofía III: argumentación fue el de desarrollar en los alumnos competencias referentes a la expresión clara y razonada de sus puntos de vista; de otorgar herramientas que les permitieran desarrollar de forma oral, pero fundamentalmente escrita, las ideas que poseen ante diversos temas de su interés. El introducir el recurso académico del ensayo argumentativo resultó fecundo, y permitió a los alumnos poner en práctica las competencias argumentativas adquiridas, mediante la defensa de un punto de vista que cada uno de ellos eligió.

\subsection{CICLO ESCOLAR 2018 - 2019}

Tras el egreso de la primera generación del IM20FS, “Juan Manuel Partida Chávez 2015 - 2018”, una generación de jóvenes acompañados de la filosofía, que la vivieron, que la sufrieron, y que en momentos odiaron, pero que, también, se hicieron conscientes de la importancia e injerencia que ésta tuvo en su formación, se dio el momento oportuno de valorar los contenidos de las asignaturas filosóficas y determinar si era pertinente realizar algún cambio. Los cambios en los temas de cada una de las asignaturas solo fueron superficiales, puntualizando algún contenido.

Así, ya en el ciclo escolar 2018 - 2019, una nueva generación de jóvenes se acercaba a terminar un ciclo acompañados de la filosofía, la cual, sin duda, nuevamente tendría injerencia significativa en su formación. En este ciclo escolar, 69 alumnos se integraron al proyecto educativo del IM20FS, en donde la filosofía, en conjunto con el resto de las asignaturas, aportaría a su formación.

Con la entrada en vigor del Plan de estudios para la educación básica (SEP, 2017b), en conjunción con logros alcanzados por algunos estudiantes de la generación “Juan Manuel Partida Chávez”, los cuales serán abordados en su momento, en el IM20FS se generó un espacio más para los contenidos filosóficos: se asignaron dos módulos para la impartición del llamado Taller de lógica: los Olímpicos. Este taller reunía a los alumnos más destacados en la asignatura de Filosofía II: lógica, para profundizar en temas lógicos, con miras en su preparación para una competencia. 


\section{OBSTÁCULOS Y LOGROS DE LA APUESTA POR LA FILOSOFÍA}

En cuanto a los obstáculos que se han encontrado por la introducción de la filosofía en el IM20FS, que no han sido poco, en las siguientes líneas se mencionarán algunos y, de ser el caso, la forma en cómo se han zanjado.

Una tentativa de obstáculo se dio con la supervisión escolar. Una vez que se asignó un supervisor escolar al IM20FS, aquél, en una visita al centro de trabajo, cuestionó la introducción de asignaturas de filosofía en el currículo, y aconsejaba que se pensara en introducir asignaturas de tipo tecnológico, que aportaran a los alumnos elementos necesarios para afrontar las nuevas tecnologías. El cuestionamiento sobre la introducción de las asignaturas filosóficas se justificaba en que éstas podrían ser el medio para una especie de adoctrinamiento político. Esto fue zanjado por las intervenciones de la dirección escolar, haciendo ver al supervisor de zona el objetivo perseguido en la introducción de la filosofía en el IM20FS. Una vez que el supervisor escolar se percató de las razones por las cuales se apostaba por la introducción de la filosofía, ofreció su apoyo al proyecto.

Otra dificultad de la impartición de la filosofía se presentó con padres de familia. Varias fueron las ocasiones en que los padres de familia manifestaron, de forma presencial o por vía telefónica, su inconformidad respecto a los temas que se abordaban en las asignaturas de filosofía, mayormente en Filosofía I: ética. Mencionaban que a ellos les había costado bastante formar un conjunto de creencias en sus hijos, como para que éstas fueran cuestionadas en la asignatura; incluso mencionaban que se intentaba cambiar el conjunto de creencias de sus hijos. Al respecto, se hizo ver a los padres de familia que, precisamente, el poner en duda, preguntarse, reflexionar e indagar era un ejercicio que se desarrollaba en la asignatura, con el objetivo de que esto se convirtiera en un quehacer habitual en los alumnos. Es decir, el objetivo de la asignatura era generar en los alumnos el hábito de cuestionarse ideas, creencias, para que ellos pudieran evaluarlas y reafirmarlas, o en su caso modificarlas, pero el objetivo no era implantar creencias: no un adoctrinamiento.

Por último, una dificultad relevante se dio con los alumnos en cuanto al cumplimiento de tareas de las asignaturas de filosofía. En las sesiones presenciales la colaboración y atención de los alumnos era la requerida, no necesitaban de mucha estimulación, las temáticas abordadas eran suficientes para que los alumnos se involucraran en las sesiones. El problema con las asignaturas de filosofía era en cuanto al cumplimiento de las actividades individuales asignadas a los alumnos para realizar en casa de forma escrita, tareas ${ }^{4}$. Los alumnos, muchas ocasiones secundadas por los padres de familia, alegaban que el

\footnotetext{
${ }^{4}$ Esta dificultad era extensiva al resto de las asignaturas impartidas en el IM20FS. En general, se presentaba una problemática de la poca disposición y capacidad de los estudiantes por la expresión escrita.
} 
cumplimiento de las tareas no era necesario ${ }^{5}$, dado que las asignaturas de filosofía no estaban reconocidas por la Secretaría de Educación Pública, que solo eran "asignaturas de relleno". Ante este tipo de pensar, la dirección escolar siempre fue enfática en que las asignaturas filosóficas formaban parte del currículo escolar, con el objetivo, en conjunto con el resto de las asignaturas impartidas en el IM20FS, de desarrollar competencias en los alumnos, y no eran "asignaturas de relleno".

En cuanto a los logros se refiere, sin duda, el más significativo ha sido la consolidación de las asignaturas filosóficas en el currículo del IM20FS, contando con el apoyo de las autoridades escolares y de la comunidad de padres de familia.

Por su parte, los estudiantes han tenido logros dignos de reconocerse en el ámbito de la filosofía. En el ciclo escolar 2017 - 2018 el IM20FS fue sede de la Eliminatoria Estatal en Nayarit de la XV Olimpiada Internacional de Lógica ${ }^{6}$. En dicha eliminatoria, realizada el día viernes 27 de abril del 2018, participaron 7 alumnos del IM20FS en la categoría de Bachillerato, siendo así los primeros alumnos de nivel secundaria en participar en el mencionado evento y compitiendo con alumnos del nivel medio superior, en los 15 años de realizarse la competencia ${ }^{7}$. También, participaron alumnos de la Unidad Académica Preparatoria No. 1, "Dr. Julián Gascón Mercado”, de la UAN, así como alumnos del Centro de Estudios Tecnológicos Industrial y de Servicios No. 100 (CETis 100) de Tepic, Nayarit. En las categorías de Licenciatura y Máster, participaron alumnos de la UAN del Programa Académico de Filosofía y de la Licenciatura en Matemáticas. Los resultados de dicha competencia fueron una razón más para justificar el quehacer de la filosofía en el IM20FS, pues los tres primeros lugares de la categoría de Bachillerato fueron obtenidos por los alumnos de secundaria: dos alumnas empataron en el primer lugar; un alumno obtuvo el segundo lugar y otro alumno obtuvo el tercer lugar. Los cuatro alumnos del IM20FS participaron en la Fase Final de la XV Olimpiada Internacional de Lógica, la cual tuvo lugar el día sábado 23 de junio del 2018 en Guanajuato, Guanajuato, México.

En el marco del XIV Encuentro de Estudiantes y Pasantes de Filosofía, organizado por el Programa Académico de Filosofía de la UAN, alumnos del IM20FS tuvieron una participación. Tres alumnos realizaron la ponencia colectiva Tres ciclos escolares de experiencias ante la filosofía, en la cual dieron a conocer su experiencia en lo correspondiente al papel de las asignaturas filosóficas en su proceso de formación desde su ingreso a la educación secundaria. Esta participación por parte de los alumnos

\footnotetext{
${ }^{5}$ No se niega que el desempeño del profesor en la impartición de las asignaturas, y el generar interés en los alumnos, haya sido deficiente, y por ello una razón más del desinterés. El profesor al que se alude, es también el autor de estas líneas.

${ }^{6}$ Dicha competencia es un esfuerzo realizado por la Academia Mexicana de Lógica (AML) para la divulgación de la lógica.

${ }^{7}$ Dado que en el reglamento de la Olimpiada Internacional de Lógica no había impedimento alguno para que alumnos de nivel secundaria participaran (OIL, 2017), se procedió a realizar las gestiones necesarias ante el Coordinador del Comité Académico y el Coordinador del Comité de Enlace.
} 
representó un esfuerzo por la divulgación de la filosofía en el proyecto educativo del IM20FS, ¡Qué mejor que la divulgación por parte de los protagonistas del proceso!

En el ciclo escolar 2018 - 2019, nuevamente, el IM20FS fue sede de la Eliminatoria Estatal en Nayarit de la XVI Olimpiada Internacional de Lógica. En esta ocasión, fueron 14 alumnos del IM20FS ${ }^{8}$ los que participaron en la categoría Preuniversitario ${ }^{9}$, donde también participaron alumnos de la Unidad Académica Preparatoria No. 1, “Dr. Julián Gascón Mercado”, y alumnos de la Preparatoria del Valle. En las categorías de Licenciatura y Máster participaron alumnos del Programa Académico de Filosofía, de la Licenciatura en Psicología y de la Licenciatura en Matemáticas de la UAN. Los resultados obtenidos al final de la competencia en la categoría Preuniversitario fueron los siguientes: el primer lugar lo obtuvo un alumno del IM20FS; tres participantes obtuvieron el segundo lugar, dos alumnos del IM20FS y una alumna de la Preparatoria del Valle ${ }^{10}$, y el tercer lugar fue ocupado por tres participantes, dos alumnas del IM20FS y un alumno de la Unidad Académica Preparatoria No. 1, "Dr. Julián Gascón Mercado"11. Estos alumnos participaron en la Fase Final de la XVI Olimpiada Internacional de Lógica, la cual tuvo lugar el sábado 18 de mayo del 2019 en la Ciudad de México.

\section{CONCLUSIÓN: DÓNDE ESTAMOS Y QUÉ FALTA POR HACER}

A pesar de las distintas dificultades, la apuesta por la filosofía en el IM20FS ha dado frutos, contribuyendo al desarrollo de competencias en los estudiantes, cumpliendo así el objetivo establecido, que incluso les han permitido incursionar en actividades de niveles educativos más allá de la educación secundaria. Es decir, la introducción de asignaturas filosóficas en educación secundaria es justificable en los documentos rectores de la educación, y la apuesta por la filosofía en el desarrollo de competencias de los estudiantes genera resultados provechosos.

La filosofía ha llegado a ser inherente al proyecto educativo del IM20FS, permitiéndole así contribuir al desarrollo de competencias en los estudiantes; la filosofía se ha convertido en parte sustancial en el proceso de formación de los alumnos.

Actualmente, se trabaja en crear un instrumento de evaluación que permita determinar el nivel de desarrollo de las habilidades y competencias en los estudiantes, propias de la introducción de las asignaturas filosóficas. Esto permitirá tomar decisiones respecto a qué cambios deben hacerse en los

\footnotetext{
${ }^{8}$ Inscritos en el Taller de lógica: Los Olímpicos, preparatorio para la competencia.

${ }^{9}$ En esta ocasión, en el reglamento de la Olimpiada Internacional de Lógica, en su artículo 18, se especificaba la posibilidad de participación de los alumnos de secundaria (OIL, 2019). De ahí, el cambio del nombre de la categoría, de Bachillerato a Preuniversitario.

${ }^{10}$ Alumna egresada del IM20FS, que en la eliminatoria del 2018 obtuviera el primer lugar. Es importante mencionar que esta alumna se integró al Taller de lógica: Los Olímpicos, preparatorio para la competencia.

${ }^{11}$ Alumno egresado del IM20FS, que en la eliminatoria del 2018 también obtuviera el tercer lugar. Igualmente, este alumno se integró al Taller de lógica: Los Olímpicos, preparatorio para la competencia.
} 
contenidos de las asignaturas, en caso de ser necesario; determinar el nivel de logro alcanzado por los estudiantes en cada ciclo escolar, y proporcionará elementos que contribuyan a refrendar la apuesta por la filosofía en el IM20FS.

Aún es necesario continuar fortaleciendo la apuesta por la filosofía en el IM20FS, continuar reuniendo más elementos que den evidencia de que no hay duda de la contribución de las asignaturas filosóficas en el desarrollo de competencias en los estudiantes, continuar con la incursión de los alumnos en espacios que van más allá del nivel de secundaria y buscar más espacios de divulgación de los resultados de incorporar la filosofía en el IM20FS. Esto con la finalidad de persuadir a otros institutos a que también apuesten por la introducción de la filosofía en su currículo.

Es claro que el camino de la introducción de la filosofía en la educación secundaria se vislumbra muy accidentado, pero, sin duda, es posible y provechoso de transitar. En el IM20FS se ha comenzado el transitar por este camino accidentado, con paso lento pero firme, dando la pauta para que otros más emprendan la travesía.

\section{AGRADECIMIENTOS}

El autor expresa su agradecimiento a la totalidad del personal del Instituto Mártires 20 de Febrero Secundaria, por su participación y entusiasmo en las actividades relacionadas con las asignaturas filosóficas, y especialmente externa su agradecimiento a la Mtra. Karla Esther González Cedano, directora del IM20FS, por el apoya, la defensa y las gestiones realizadas en favor de la introducción, y permanencia, de la Filosofía en el proyecto educativo del Instituto. 


\section{REFERENCIAS BIBLIOGRÁFICAS}

Amor, J. (2003). "La enseñanza del análisis lógico", en Campirán Salazar, Ariel, compilador, La razón comunicada ll. Materiales del taller de didáctica de la lógica, México: TDL - UX - AML - Torres Asociados, pp. 39-58.

Beauchamp, T. y Childress, J. (1999). Principios de Ética Biomédica, México: Masson S. A.

Campirán, A. (2003). "Pensamiento creativo en el aprendizaje de la lógica", en Campirán, A., compilador. La razón comunicada ll. Materiales del taller de didáctica de la lógica, México: TDL - UX - AML Torres Asociados, pp. 59-69.

Centro de Escritura Javeriano. (2019). Normas APA, sexta edición. Cali, Colombia: Pontificia Universidad Javeriana, seccional Cali. https://www.javerianacali.edu.co/sites/ujc/files/normas_apa_revisada_y_actualizada_mayo_2019.pdf

Collingwood, R. (1954). "Pregunta y respuesta", en Autobiografía, México: Fondo de Cultura Económica, pp. 37-50.

Deaño, A. (1985). Introducción a la lógica formal, España: Alianza Universidad.

Diario Oficial de la Federación (DOF). (1982). Acuerdo por el que se establece la organización y funcionamiento de las Escuelas de Educación Secundaria. Acuerdo 98. México: Gobierno de México. https://www.dof.gob.mx/index.php?year=1982\&month=12\&day=07

DOF. (2013). Decreto por el que se reforman los artículos 3o. en sus fracciones II, VII y VIII; y 73, fracciones XXV, y se adiciona un párrafo tercero, un inciso d) al párrafo segundo de la fracción II y una fracción IX al artículo 3o. de la Constitución Política de los Estados Unidos Mexicanos. México: Gobierno de México. https://www.dof.gob.mx/nota_detalle.php?codigo=5288919\&fecha=26/02/2013

DOF. (2019). Decreto por el que se reforman, adicionan y derogan diversas disposiciones de los artículos 3o., 31 y 73 de la Constitución Política de los Estados Unidos Mexicanos, en materia educativa. México: Gobierno de México. https://www.dof.gob.mx/nota_detalle.php?codigo=5560457\&fecha=15/05/2019

Eemeren, F., Grootendorst, R. y Snoeck, F. (2006). Argumentación: análisis, evaluación, presentación, Argentina: Biblos.

Escobedo, M. (1995). Lógica formal. La idea, el juicio, el raciocinio, México: Trillas.

Estanislao, J., Álvarez, J. y Guerra, C., (editores). (2015). Hacer Filosofía con niños y niñas. Entre educación y filosofía, Chile: Universidad de Valparaiso.

Fernández, M. y Villegas, L. (2011). Lógica matemática I: lógica proposicional, intuicionista y modal, México: Universidad Autónoma Metropolitana.

Ferrater, J. (1957). ¿Qué es la lógica?, Argentina: Editorial Columbia.

Garrido, M. (editor). (1989). Lógica y Lenguaje, Madrid, España: Tecnos.

Garrido, M. (2001). Lógica simbólica, España: Tecnos. 
Herrera, A. y Torres, J. (2007). Falacias, México: Torres Asociados.

Instituto Mártires 20 de Febrero Secundaria. (2019). Reglamento Interno Escolar.

Ciclo escolar

https://onedrive.live.com/?authkey=\%21 AADUX\%5FFag3ppnRY\&cid=246252FD24BA36D8\&id=246

252FD24BA36D8\%211357\&parId=246252FD24BA36D8\%21450\&o=OneUp

INEE. (2015). Panorama Educativo de México 2014. Indicadores del Sistema Educativo Nacional. Educación básica y media superior, México: INEE. https://www.inee.edu.mx/wpcontent/uploads/2018/12/P1B113.pdf

INEE. (2016). Panorama Educativo de México 2015. Indicadores del Sistema Educativo Nacional. Educación básica y media superior, México: INEE. https://www.inee.edu.mx/wpcontent/uploads/2019/01/P1B114.pdf

Katayama, R. (2011). Lógica, Perú: Editora Lealtad.

Lengyel, O. (2006). Los Hornos de Hitler, México: DIANA.

Lozano, M. y Pérez, A. (2016). Manual de lógica elemental, México: Trillas.

Marraud, H. (2013). ¿Es Lógic@ ? Análisis y evaluación de argumentos, España: Cátedra.

Morado, R. (1999). “¿Qué debe saber de lógica una persona educada?”, en Morado, R., (compilador), La razón comunicada. Materiales del taller de didáctica de la lógica, México: TDL - UX - UV - AML Torres Asociados, pp. 7-15.

Olimpiada Internacional de Lógica (OIL). (2017). Reglamento general de la Olimpiada Internacional de Lógica.

http://academiamexicanadelogica.org/sites/default/files/convocatorias/Reglamento\%20general\%20de\%2 0la\%20Olimpiada\%20Internacional\%20de\%20L\%C3\%B3gica_0.pdf

OIL. (2019). Reglamento General Unificado de la Olimpiada Internacional de Lógica. https://drive.google.com/file/d/1E1BQuEfjGrkVcwhw6Rt1EqGGB5Bf13vt/view

Oscos, M., (coordinadora editorial). (2010a). “Textos argumentativos”, en Enciclopedia de conocimientos fundamentales vol. 1, México: UNAM - Siglo XXI, pp. 80-104.

Oscos, M., (coordinadora editorial). (2010b). "El ensayo", en Enciclopedia de conocimientos fundamentales vol. 1, México: UNAM - Siglo XXI, pp. 322-353.

Pacheco, L. (2006). Metodología de la investigación. La elaboración del proyecto, Tepic, Nayarit, México: Universidad Autónoma de Nayarit.

Platón. (1985). Diálogos 1. Apología, Critón, Eutifrón, Ion, Lisis, Cármides, Hipias menor, Hipias mayor, Laques, Protágoras, Madrid, España: Gredos.

Platón. (1988). Diálogos 4. República, Madrid, España: Gredos. 
Rachels, J. (2006). Introducción a la filosofía Moral, México: Fondo de Cultura Económica.

Schulman, M. (s/d). El amor es una falacia. http://decsa.med.uchile.cl/wpcontent/uploads/AmorComoFalacia.pdf

Secretaría de Educación Pública (SEP). (2011). Plan de estudios, México: Secretaria de Educación Pública. https://www.gob.mx/cms/uploads/attachment/file/20177/Plan_de_Estudios_2011_f.pdf

SEP. (2016). El modelo Educativo 2016. El planteamiento pedagógico de la Reforma Educativa. México: Secretaría de Educación Pública. https://www.gob.mx/cms/uploads/attachment/file/114501/Modelo_Educativo_2016.pdf

SEP. (2017a). Carta los fines de la educación en el siglo XXI, México: Secretaría de Educación Pública. https://www.gob.mx/cms/uploads/attachment/file/207276/Carta_Los_fines_de_la_educacio_n_final_03 17_A.pdf

SEP. (2017b). Aprendizajes clave para la educación integral. Plan y programas de estudio para la educación básica, México: Secretaria de Educación Pública. http://www.sep.gob.mx/work/models/sep1/Resource/10933/1/images/Aprendizajes_clave_para_la_educ acion_integral.pdf

Silva, M. (2016). "Competencias de estudiantes iberoamericanos al finalizar la educación secundaria alta”. Perfiles Educativos, XXXVIII (152), $\quad$ pp. 88 - 108. http://www.perfileseducativos.unam.mx/iisue_pe/index.php/perfiles/article/view/57598/51081

UNESCO. (2011). La filosofía. Una escuela de libertad, México: UNESCO - UAM Unidad Iztapalapa. http://www.ofmx.com.mx/documentos/pdf/Filosofia_unaescueladelalibertad_UNESCO.pdf

Universidad Autónoma de Nayarit (UAN) y SETUAN. (2018) Contrato Colectivo de Trabajo 2018 2019. Sindicato de Empleados y Trabajadores de la Universidad Autónoma de Nayarit. http://www.setuan.org.mx/docs/contrato.pdf

Tugendhat, E., Vicuña, A. y López, C. (2010). El libro de Manuel y Camila. Diálogos sobre ética, España: Gedisa.

Suppes, P. (1973). Introducción a la lógica simbólica, México: CECSA.

Silva, B. y Juárez, J. (2013). Manual del modelo de documentación de la Asociación de Psicología Americana (APA) en su sexta edición, Puebla, México: Universidad Popular Autónoma del Estado de Puebla. http://online.upaep.mx/LPC/online/apa/APAimp.pdf

Vega, L. (2013). La fauna de las falacias, España: Trotta.

Vega, L. (2016). Introducción a la teoría de la argumentación. Problemas y perspectivas, Lima, Perú: Palestra.

Weston, A. (2013). Las claves de la argumentación, México: Ariel. 